\section{Cielos australes, tormentas sociales y pestilencias: astronomía y meteorología en el debate parlamentario argentino, 1869-1872}

\section{Southern skies, social storms and pestilences: astronomy and meteorology in Argentine parliamentary debates, 1869-1872}

\section{Marina Rieznik ${ }^{i}$}

' Investigadora del Consejo Nacional de Investigaciones Científicas y Técnicas en el Instituto de Estudios Sociales de la Ciencia y la Tecnología/ Universidad Nacional de Quilmes.

$$
\text { Bernal - Argentina }
$$

orcid.org/0000-0002-9710-1486

marinarieznik@gmail.com

\section{Andrea Comercii}

ii Docente, Departamento de Materias Básicas/

Universidad Tecnológica Nacional.

Buenos Aires - Argentina

orcid.org/0000-0001-9794-0258

andreacomerci@yahoo.com.ar

Recebido em 24 jul. 2018.

Aprovado em 3 jan. 2019.

http://dx.doi.org/10.1590/S0104-59702020000200007
RIEZNIK, Marina; COMERCI, Andrea. Cielos australes, tormentas sociales y pestilencias: astronomía y meteorología en el debate parlamentario argentino, 1869-1872. História, Ciências, SaúdeManguinhos, Rio de Janeiro, v.27, n.2, abr.-jun. 2020, p.431-446.

Resumen

Entre 1869 y 1872 se debatieron los proyectos de creación del Observatorio Nacional Argentino y de la Oficina Meteorológica Argentina. Las propuestas de financiamiento de nuevas instituciones nacionales, así como su articulación con las políticas públicas, estaban atravesadas por conflictos propios de la etapa de construcción del Estado. Oponiéndose a los proyectos elevados al Congreso Nacional, algunos sectores argumentaban recursos escasos. El presidente, Domingo Sarmiento, los acusaba de ser aliados de un gobierno anterior, considerado como "bárbaro". No obstante, mostraremos que quienes defendían los proyectos, consideraban también inadmisible un gasto elevado, remarcaban que era poco y lograban su aprobación articulando el discurso con políticas entonces en curso que apuntaban a otras inquietudes como la educación, la inmigración y las epidemias.

Palabras clave: meteorología; astronomía; Argentina; siglo XIX; Parlamento.

Abstract

From 1869 to 1872 , there was debate about bills to create the Observatorio Nacional Argentino and the Oficina Meteorologica Argentina. The proposed funding for these new national institutions, as well as their connection to public policies, were riven by conflicts inherent to that phase of the construction of the State. Some sectors opposed the bills before the Congreso Nacional, arguing that resources were scarce. President Domingo Sarmiento charged that they were allies of the previous government, which he described as "barbarous." This article shows that the bills' supporters stressed that they were low-cost. They achieved passage of the bills by linking their discourse with contemporary policies aimed at other concerns, such as education, immigration and epidemics.

Keywords: meteorology; astronomy; Argentina; nineteenth century; Parliament. 
E n la Argentina, entre 1869 y 1872, durante la presidencia de Domingo Faustino Sarmiento, se debatieron en las cámaras parlamentarias dos proyectos de leyes para crear y financiar el Observatorio Nacional Argentino y la Oficina Meteorológica Argentina. En 1870, el ministro de Justicia, Culto e Instrucción Pública, Nicolás Avellaneda, invitó formalmente al astrónomo norteamericano Benjamin Gould a organizar y dirigir el observatorio que fue inaugurado en 1871 en los alrededores de la ciudad de Córdoba. Un año después, Gould propuso crear una Oficina Meteorológica Nacional, que él coordinaría gratuitamente desde el mismo edificio.

Las primeras historias sobre el Observatorio Nacional fueron escritas por la historiografía tradicional de las ciencias e incorporadas en las narrativas institucionales. Basándose en los discursos de las élites, mostraban la investigación desarrollada en ese espacio como consecuencia de un proyecto nacional de construcción del Estado moderno que habría sentado las bases de la ciencia argentina. Los trabajos científicos impulsados en el observatorio se señalaban como logros de una élite, personificada en hombres como Sarmiento o Avellaneda. La ciencia argentina avanzaba durante décadas, caracterizadas como gloriosas, dejando atrás el período violento dominado por Rosas, en el que las luchas intestinas, "el caudillismo", y "la tiranía" habían impedido el "florecimiento científico". Recién con la caída de Rosas, en 1852, se empezaría a modificar el telón de fondo del espíritu colonial, cuando no había ciencia en el río de la Plata, sino ciencia de las metrópolis que usaban para ello el territorio americano (Babini, 1954, 1986; Chandler, 1896; SCA, 1979). Hace ya más de una década, en lugar de retomar acríticamente los discursos inaugurales sobre los observatorios pronunciados por los miembros de las élites, propusimos integrarlos con diferentes fuentes, mostrando los intereses internacionales que existían por detrás de estas instituciones financiados por el Estado argentino a finales del siglo XIX. Lo hicimos desde una historiografía centrada en el análisis de las prácticas científicas y de los procesos de trabajo en los observatorios, con una metodología que retomaba líneas de análisis propuestos por la epistemología histórica. Asimismo, valorizamos los aportes de autores, como Pyenson (1985), que habían mostrado la importancia de las redes internacionales de trabajo científico entretejidas en suelo local, y pudimos verificar que eran claves para entender la fundación y dinámicas del observatorio. Solo atendiendo a las necesidades de esas redes de astrónomos por coordinar, homogeneizar y mecanizar los procesos de trabajo en los observatorios de la época desde los dos hemisferios, pudimos ir mostrando cómo las necesidades locales se habían ido transformando y acoplando en el observatorio. Así, llegamos a dar cuenta de la formación de astrónomos argentinos en las primeras décadas del siglo XX, señalando necesidades locales y líneas de investigación que de ninguna manera existían al inicio del proceso (Rieznik, 2011). Nuestros análisis forman parte de un conjunto de nuevas perspectivas abiertas en la historiografía de las ciencias de las últimas décadas en la Argentina, que sitúa el desarrollo científico de fines del siglo XIX en un contexto más amplio de conflictos entre diversos intereses locales e internacionales, propios de la etapa de formación del Estado nacional.

Por otro lado, desde la década de 1980, una amplia y variada vertiente de renovación de la historiografía argentina fue exponiendo diversas incongruencias de la historiografía anterior, que se había centrado en mostrar la linealidad entre las ideas de la élite, los planes 
orquestados a partir de ellas y la construcción del Estado nacional hacia fines del siglo XIX. Así, se señalaron convergencias, mediaciones y tensiones de los discursos e imaginarios de la unidad nacional - entre ellos los que se autoproclamaban avanzando de la "barbarie" a la "civilización" - respecto de la heterogeneidad de los procesos de formación estatal. Fueron diversas las áreas enfocada por quienes pusieron en tela de juicio esa supuesta linealidad. Analizaron la estructura de la propiedad agraria, las variaciones de las economías regionales, las estructuras comerciales, las formas jurídicas locales, las movilizaciones o reclutamientos políticos y militares, entre otras cuestiones. ${ }^{1}$ Abundaron entonces estudios históricos que iban más allá de la consideración literal de los discursos de las élites, ya sea para denostarlas o ennoblecerlas.

En el marco historiográfico de cuestionamiento a las narrativas clásicas, retomaremos una línea de interpretación que está presente también en trabajos como los de Podgorny y López (2008) sobre el Museo de La Plata a fines del siglo XIX. Las autoras remarcaron que la investigación científica requería planes institucionales de largo plazo y que el presupuesto nacional se mostraba escaso para comprometerse en ellos, aunque también generoso si se trataba de obras que pudieran exponer la grandeza nacional. Asimismo, mostraron que las políticas de los representantes del Estado respecto al financiamiento de los museos muchas veces estaban justificadas en última instancia porque sustentaban instituciones educativas, mientras que parecían mucho menos los dispuestos a atrincherarse detrás de eventuales líneas de investigación como necesarias (Podgorny, 2009; García, 2010).

Coincidiendo con los enfoques mencionados, mostraremos que muchas veces los argumentos desplegados por quienes propusieron las dos leyes en cuestión tenían poco que ver con los resultados que se esperaban de los trabajos científicos que desarrollarían. A veces, como ocurriría en el caso de los museos, se trataban a las instituciones como símbolos de grandeza nacional o como refuerzos de instituciones educativas. Otras veces, se apelaba a encastres con políticas económicas, inmigratorias, higiénicas. Asimismo, tanto defensores como opositores de los proyectos subrayaban que era importante minimizar el gasto que significarían las instituciones para las arcas del tesoro. Creemos que la revisión de los debates parlamentarios, en combinación con otras fuentes, publicaciones periódicas, cartas y bibliografía secundaria, nos permiten reforzar nuestra hipótesis: la apelación a lo exiguo del gasto necesario, así como la debilidad en la explicitación de los objetivos de investigación de las instituciones creadas, no eran episodios coyunturales, sino una estrategia más general del Poder Ejecutivo respecto a la fundación de instituciones científicas.

Hace unos años, mostramos por primera vez los detalles de los debates parlamentarios de la legislación y asignación de las primeras partidas para el observatorio (Rieznik, 2011). En el apartado siguiente, retomaremos parte de ese análisis para compararlo con los debates en torno a la financiación de la Oficina Meteorológica Nacional, que expondremos en los apartados subsiguientes. Entre el debate de una ley y el de la otra, el punto sobre la nimiedad del gasto, tópico de la primera discusión, se consolidó como parte de la escritura del proyecto siguiente. El contexto que debe tenerse en cuenta concierne a las pugnas por la asignación de presupuestos para instituciones estatales, y a las disputas de sectores que proponían prioridades diferentes entre las urgencias sociales que debían atenderse. 


\section{El debate parlamentario sobre el observatorio}

Aunque los debates parlamentarios habían sido dejados al margen por la historiografía tradicional, era llamativo que nadie hubiera dado cuenta de los mismos, ya que sus trazos se encontraban inclusive en un discurso ampliamente retomado; el de Sarmiento (1871, p.1) en la inauguración del observatorio:

Es anticipado se dice, un Observatorio en pueblos nacientes y con un erario ó exhausto ó recargado ... Los que hallan inoportuno un Observatorio Astronómico, nos aconsejan lo que Rosas practicaba, lo que Felipe II legó a sus sucesores, y nos separa por fin de la especie humana, en todos los progresos realizados mediante el estudio de las ciencias naturales, desde el renacimiento hasta nuestros días, en el resto de Europa y en los Estados Unidos ... ¿Cuánto necesitamos nosotros, los rezagados de cuatro siglos, para alcanzar en su marcha a los pueblos que nos preceden? El Observatorio Astronómico Argentino es ya un paso dado en este sentido.

La historiografía había retomado este discurso donde la construcción del observatorio aparecía ligada a la historia del progreso en la Argentina. El proceso habría sufrido un retroceso en manos del gobierno de Juan Manuel de Rosas. En ese sentido, el observatorio era puesto como símbolo del avance de los procesos de modernización y unidad nacional tras la caída del "tirano" en 1852, una muestra de la capacidad estatal en relación con la transformación del orden social.

Como ya hemos señalado, Sarmiento no ocultaba que sus discursos eran textos "de batalla" y que con ellos pretendía poner al descubierto a quienes, agazapados en la trinchera opuesta, según su entender, promovían las guerras interprovinciales. Sin embargo, la historiografía tradicional presentó estos discursos como relatos objetivos del devenir del enorme esfuerzo hecho por la ciencia en favor de la unidad nacional, dejando en la sombra los argumentos de quienes se oponían a la fundación del observatorio y toda disputa que pudiera nublar la idea del éxito de la supuesta planificación central del desarrollo de las investigaciones científicas (Rieznik, 2011). Sin embargo, los estudios historiográficos mencionados en la introducción mostraron que, tras la caída de Rosas, los enfrentamientos persistían entre los gobiernos provinciales, y entre los mismos y el Estado central en construcción, en torno a cuestiones como la distribución de la renta, el control de los órganos de coerción y la delimitación de áreas territoriales. En ese sentido, las oposiciones a Sarmiento, que persistían en algunas provincias, no eran el reflejo de la continuidad de una "batalla" entre unitarios y federales, sino la manifestación de la no linealidad de los procesos de unidad nacional en los que no se agrupaban fácilmente a rosistas federales a un lado y unitarios al otro.

El proyecto para financiar el observatorio había sido inicialmente sometido a debate en la sesión del 3 de septiembre de 1869. Santiago Cortinez inició la polémica argumentando que votaría en contra. El diputado de la provincia de San Juan había ocupado distintos lugares en las luchas facciosas de la época y se había alineado con el ejército unitario unos años atrás. Difícilmente podía verse en él a un representante de las políticas de Rosas, más bien era uno de los agentes del heterogéneo proceso de unidad nacional. No es un dato menor que Sarmiento terminara nombrando a Cortinez como ministro de Hacienda en 
1874; y que luego continuara en el cargo y fuera también ministro de Avellaneda. En 1869, cuando el diputado argumentaba en contra de la iniciativa, afirmaba que el estado de la República, su educación, su progreso material, eran muy rudimentarios y que existían muchas necesidades preferibles a esa; la nación tenía necesidades urgentes, financiar el observatorio era "como exigir a un niño que careciese de educación básica, que estudiara las combinaciones más difíciles del Aljebra" (Diario..., 3 sept. 1869, p.237). La posición de Cortinez en la Cámara no parecía tan descabellada a los ojos de Avellaneda. Por eso, contestó que el gasto no sería permanente, puesto que la suma tan abultada se debía a que era necesaria para la construcción del edificio y para la instalación de instrumentos; luego bastarían con dos o tres mil pesos para su funcionamiento. Lo que se argüía contemplaba las razones de Cortinez; el gasto en ciencia sería nimio para las arcas del tesoro.

Por otro lado, al igual que el presidente, Avellaneda asociaba las necesidades del desarrollo científico a una de las trincheras de las disputas locales. Explicaba que, a diferencia de lo que ocurría en el hemisferio Norte, en toda América del Sur faltaba el trabajo de catalogar las estrellas, y remarcaba que esta carencia era sentida desde hacía mucho tiempo. Según Avellaneda, el "progreso civilizado" solicitaba a los pueblos de América del Sur que dejasen de malgastar su dinero en guerras y que se dedicaran a fundar observatorios. El argumento parecía volcado a generar la empatía de los antirosistas que aún se oponían al proyecto, más que a polemizar con defensores de las políticas de Rosas.

Finalmente, agregaba Avellaneda que la nación que había acudido en primer lugar a la solicitud había sido Chile, cuyo gobierno tenía un observatorio y no estaba en condiciones muy diferentes a las nuestras. ${ }^{2}$ Por otro lado, el ministro destacaba que el futuro director del observatorio podría desempeñar una clase en la Universidad de Córdoba y que por tanto su sueldo sería "doblemente útil" (Diario..., 3 sept. 1869, p.238).

El 2 de octubre del mismo año, en la Cámara de Senadores, quien argumentó en contra del proyecto, apelando a idénticos motivos que Cortínez, fue el señor Plácido Sánchez de Bustamante. El senador era el representante de una familia que dominaba la vida política de Jujuy, repartiéndose los cargos públicos, políticos y admistrativos de la provincia. Si bien unos años después apoyaría a Bartolomé Mitre contra Avellaneda, cristalizando parte de los conflictos en marcha; era asimismo integrante de un clan perseguido por Rosas, que había concentrado su poder con la caída de este (Paz, 2003). Entonces Avellaneda comenzó su defensa con el esquema presentado en la Cámara de Diputados, centrado en que se requeriría solo de una "pequeña cantidad" para el establecimiento (Diario..., 2 oct. 1869).

Las limitaciones de los defensores del proyecto para poder dar cuenta de las actividades que se desarrollarían en la institución eran notables. El ministro explícitamente admitía no saber explicar cuáles serían las utilidades prácticas reportadas para nuestros pueblos por la instalación de un observatorio que se dedicara a catalogar estrellas. Intentando alivianar la incertidumbre, argumentaba que los pueblos más adelantados habían invertido tanto en observatorios, que ello no podía ser en vano. Insinuaba que existía un abanico de utilidades prácticas que la nación se aseguraría con esa fundación, aunque no podía explicitarlas.

Cuando el ministro ya estaba trabado en este punto, no pudiendo explicar la utilidad de la institución, asegurando que prescindiendo de los resultados científicos, nos traería notoriedad, adentrándose en el terreno de las cartas de presentación para los encuentros 
internacionales y en el de cómo atraer a sabios para que nos prestasen sus luces, intervino Bartolomé Mitre, entonces senador por Buenos Aires. Advertía que en realidad la utilidad del observatorio no debía asociarse a la catalogación de estrellas. La tarea importante, según Mitre, en referencia a los catálogos, ya la habían realizado los EEUU en Chile, en un lapso relativamente breve. Sin embargo, se habían formado astrónomos chilenos bajo la dirección de sabios extranjeros y ese debía ser el ejemplo a seguir. No se trataba de misteriosos provechos prácticos, ni de credenciales ante los pueblos avanzados, sino de la formación de astrónomos en suelo local. Mitre sugería que se orientara el dinero a la formación de una escuela astronómica en la institución. Avellaneda confirmaba que Gould daría clases en la Universidad (Rieznik, 2011). Finalmente, la ley n.484 del Presupuesto General de la Nación para 1870 aprobaba un monto anual total de aproximadamente 14 millones de pesos fuertes y destinaba 31.980 pesos fuertes a la construcción del edificio (Diario..., 8 sept. 1869, p.1245).

\section{La propuesta de creación de la oficina meteorológica}

Gould había nacido en Boston en 1824. Había estudiado matemática y física en Harvard College, y se había formado con Friedrich Argelander en Göttingen. En 1870 llegó a la Argentina para dirigir el Observatorio Nacional. ${ }^{3}$ A pesar de los argumentos esgrimidos en el Congreso, Gould nunca dio clases en la universidad. El plan que tenía en mente, tal como mostramos en nuestros trabajos sobre el tema, se apoyaba en redes cuyos planes de trabajo tenían poco que ver con la enseñanza y con la formación de astrónomos locales (Rieznik, 2011). Se dedicó sobre todo a la construcción de catálogos y mapas celestes del hemisferio austral. Creemos que la experiencia de Gould en Albany marcó su aversión personal a la idea de que la administración de recursos y el ejercicio de funciones en distintas reparticiones estatales pudieran ayudar al desarrollo astronómico. En 1856, en los EEUU, al volver de un entrenamiento con astrónomos germanos, había emprendido la dirección en el Observatorio Dudley y vivido una experiencia tormentosa. Los opositores de Gould en EEUU opinaban que había sido ineficaz en las tareas asignadas, que malversaba fondos y que se resguardaba en el puesto que tenía bajo auspicios del Estado. A uno y otro lado de la disputa era señalado el principal problema de los planes en marcha: la conexión entre la Coast Survey, es decir el servicio hidrográfico, y el Observatorio de Dudley y la consecuente falta de claridad respecto a las tareas que correspondían a cada institución (Wilder, 1858). Gould sostenía que el problema había sido trabajar para la Coast Survey al mismo tiempo que emprendía la dirección del Observatorio de Dudley lo que habría hecho imposible volcar la energía que requería el verdadero trabajo científico (Wilder, 1858).

Después de un año de trabajo en la Argentina, el 24 de mayo de 1871, Gould elaboró para Avellaneda un primer informe sobre las actividades del observatorio. En el mismo, además de dar cuenta de los trabajos realizados en el observatorio, expresaba la necesidad de labores sistemáticas de observaciones meteorológicas en toda la República. Afirmaba que servirían de auxilio para las observaciones astronómicas y que además serían de utilidad para fines económicos. Hacía una referencia retórica a la utilidad económica que redundaría al tener conocimiento de las particularidades climáticas del país y, aunque no 
avanzaba en esta especificidad, detallaba los primeros pasos para orientar los trabajos de la meteorología local. Gould (1872, p.10) afirmaba:

El otro modo no menos obvio, de utilizar las fuerzas del Observatorio, consistiría en la organización y mantenimiento de un sistema de observaciones meteorológicas coordinadas, que podrán de manifiesto las peculiaridades climatéricas y leyes atmosféricas que dominen en estas regiones: relaciones y leyes bien diferentes de las de otros países, y que son por lo tanto del mayor interés teórico y práctico ... Por otra parte, un sistema de observaciones meteorológicas coordinadas y ejecutadas bajo un plan uniforme en los diferentes puntos de la república, con concepto a formar un todo concordante, sería de la mayor importancia, no sólo en el orden científico, sino también en el orden económico.

En un informe anual posterior fechado el 15 de marzo de 1872, enviado a Avellaneda, Gould (1872, p.38) insistía sobre la falta de sistematización en el país de observaciones meteorológicas y resaltaba el rol clave que el Observatorio Nacional podría tener para coordinarlas ante la carencia de otro tipo de iniciativas al respecto:

No entraba en mi plan original incluir trabajos de este género [meteorológico] en la actividad de nuestro Observatorio, puesto que ellos salen fuera de la esfera de la ciencia a la que está dedicada nuestra institución. Sin embargo, después de una corta residencia en el país llegué a persuadirme de las singularidades de sus fenómenos atmosféricos, y de lo importante que sería obtener observaciones climáticas regulares y coordinadas en varias partes de la República.

Recordar la experiencia que había tenido Gould antes de llegar a la Argentina es fundamental para entender porque no pedía simplemente un aumento presupuestario para las tareas que proponía coordinar, sino que solicitaba destinar una partida diferente para hacer observaciones meteorológicas y proponía crear una institución distinta. El reconocimiento de la Oficina Meteorológica por ley implicaba más riesgos, en términos de burocracia, para aprobar los fondos para la nueva dependencia; suponía someterse nuevamente a un debate en el Parlamento sobre la necesidad de impulsar investigaciones científicas). No obstante, implicaba asegurar la autonomía de la administración de recursos del Observatorio Astronómico. Por el contrario, pedir un aumento de fondos para tareas meteorológicas, que él explícitamente se comprometía a coordinar, y hacerse cargo de los instrumentos en cuestión desde la misma institución podía traer complicaciones que terminasen afectando la tarea principal a la que quería abocarse: la construcción de catálogos y mapas celestes. Es en este contexto que se entiende la advertencia que hacía Gould en 1872, cuando impulsaba la ley de creación de la Oficina Meteorológica Argentina (Bernaola, 2001; Gould, 1888). Entonces afirmaba que en distintos lugares se habían unido las funciones de un observatorio astronómico y físico, pero que la experiencia se había encargado de demostrar que la mencionada unión había sido, en la gran mayoría de los casos, esencialmente perjudicial al departamento astronómico. Por eso, una vez creada la institución, el director se empeñaría en mantener esas dos ramas de investigación científica enteramente separadas "una máxima que espero será siempre conservada en el observatorio argentino" (Gould, 1878, p.129). 
Lo cierto es que Sarmiento se hizo eco de esta solicitud de Gould, de crear una oficina independiente y el 18 de agosto de 1872 redactaba la carta que presentó junto al proyecto de ley en el Congreso Nacional. En la carta, además de las razones de orden científico, implícitamente aludidas de manera general también por Gould, Sarmiento esgrimía motivos de orden económico para la creación de la Oficina Meteorológica Argentina y avanzaba en precisiones que Gould no había dado. Repetía entonces la idea frecuente de que el conocimiento del clima de toda la República redundaría en la posesión de "datos más claros para la mejor dirección económica de nuestra agricultura" (Diario..., 14 sept. 1872, p.215). Dicha actividad se había constituido en un tema vertebral de su discurso; según Botana (1996, p.33), "Sarmiento fue un discípulo lejano de [Thomas] Jefferson, guiado por la convicción de que la agricultura estaba entrañablemente ligada con la civilización republicana", quien se dedicara a esta actividad se reservaría la virtud genuina para el abastecimiento de una república con bienes materiales y espirituales, "para colmar ese espacio con abundancia de productos y con ciudadanos autosuficientes". La idea sostenida por Sarmiento de la agricultura como factor de desarrollo de la nación quedó registrada inclusive en sus escritos literarios. En Facundo, Sarmiento evocaba a la actividad, convencido de que el desarrollo de ésta no solo posibilitaba la subsistencia de un pueblo, desde el punto de vista alimenticio, sino que, apoyada en la variable comercial, lograba el progreso de una población y la salida de la "barbarie" (Palcos, 1945). Coherente con su temprana línea de interpretación, Sarmiento, el 17 de julio de 1870, había impulsado en el Senado la apertura del Departamento de Agricultura (Barsky, Gelman, 2001). Un año más tarde, el presidente asociaba el pedido de Gould con esta política; otro indicio de que las partidas presupuestarias se aprobaban, no apelando al sostenimiento de la investigación científica per se sino solo en tanto se presentaran como complemento de apoyo a políticas estatales ya aprobadas.

\section{Meteorología y discusiones parlamentarias}

El 14 de septiembre de 1872, se llevó a cabo el debate en torno a la creación de la Oficina Meteorológica Argentina en la Cámara de Senadores. El proyecto del Poder Ejecutivo postulaba en su artículo primero: "Créase una Oficina Meteorológica Argentina, con el objeto de formar un sistema regular de observaciones meteorológicas en toda la república, y cuya residencia será establecida en el punto que el Poder Ejecutivo estimare conveniente" (Diario..., 14 sept. 1872, p.215). El Ejecutivo ya había adelantado que le parecía conveniente que fuera en el observatorio y la legislación y asignación del presupuesto fueron objeto de polémicas entre los representantes de los poderes Legislativo y Ejecutivo. La comisión para tratar el proyecto referido fue conformada por los senadores Wenseslao Colodrero, miembro informante de la Comisión, y los señores Juan Llerena y Daniel Araoz.

Colodrero, senador por Corrientes, fue el encargado de fundamentar el proyecto, resaltando la importancia y necesidad de la creación de la Oficina Meteorológica. Sin más detalle, pasaba a leer el proyecto de ley enviado por Sarmiento. Asimismo, leía una carta de presentación del proyecto afirmando que "el poder ejecutivo piensa que es inútil encarecer ante la ilustración de V.H.: la importancia, teórica y práctica, científica y económica de 
estos estudios, que se relacionan además con intereses valiosos y visibles" (Diario...14 sept. 1872, p.215). Es decir, se retomaban las apelaciones de Gould, y no sólo no se explicitaban cuáles serían los beneficios en todos los órdenes mencionados, sino que se sugería que solo alguien con pocas luces podría no verlos.

Frente a semejante advertencia, el señor Llerena, senador por San Luis, a pesar de manifestar que el proyecto "era vago", afirmaba que daría su voto positivo. La línea divisoria propuesta por Sarmiento se invertía en este caso: mientras que este senador, que sí había tenido vínculos con las organizaciones rosistas, votó a favor del proyecto, Araoz, enfrentado a dichos círculos políticos en el pasado, votó en contra. El senador por Jujuy esgrimía que "abstractamente considerado el asunto, era aceptable en teoría, no así en cuanto a la cuestión práctica" ya que demandaría una cantidad de dinero que bien podía utilizarse en algo más provechoso como, por ejemplo, el rescate de los "cautivos que existían en poder de los salvajes" y otros tendientes a pagar "deudas sagradas de la nación" (Diario..., 14 sept. 1872, p.218). Volvía salir a la luz el argumento de Bustamante oponiéndose a la creación del Observatorio Astronómico, planteando que las arcas del tesoro no estaban para gastos no urgentes, cuando existían otros más perentorios. No era casual, Aráoz integraba también la familia Bustamante por línea materna y sus intereses eran convergentes.

Avellaneda tomó entonces la palabra para dar respuesta a lo expresado por Araoz. No lo hizo atacando el argumento, sino incorporándolo, subrayando el bajo costo que exigía la fundación de la institución. La maniobra era una estrategia reiterada del ministro y surtiría mayor efecto si se consideraba que se pedía una quinta parte de lo que se había solicitado para el observatorio, tanto para la instalación inicial como para el funcionamiento de rutina. Más aún, en este caso, la nimiedad del gasto se remarcaba en el propio proyecto cuando se establecía que el trabajo del director para esta oficina sería gratuito. La carta del Ejecutivo también se ocupaba del asunto aclarando que "Los colegios que han sido dotados de gabinetes de Física, poseen algunos de los instrumentos más indispensables para la práctica de la meteorología y el completarlos en estos establecimientos ó proporciónalos á los demás, es cuestión que no demanda gastos de importancia" (Diario..., 14 sept. 1872, p.216). Además, el propio proyecto de ley se había encargado de responsabilizar a los profesores de los colegios, escuelas nacionales y otros voluntarios, que colaborarían gratuitamente en la red de observaciones por los eventuales deterioros o pérdidas de instrumentos. ${ }^{4}$ Avellaneda agregaba que además el gasto estaría vinculado con: "el conocimiento y estudio de las causas producentes de las epidemias que contenía la inmigración. ... el estudio de la atmósfera es esencial, si se quería alejar aquellas" (Diario..., 14 sept. 1872, p.216).

Detengámonos en el argumento sobre la prevención de enfermedades contenida en este fragmento y en la relación entre salud y meteorología mencionada. Debe tenerse en cuenta que Avellaneda amalgamaba en esta cita argumentos ligados a las teorías contagionistas, que consideraban que las epidemias de la época se estaban transmitiendo entre personas ("epidemias que contenía la inmigración"), con otros vinculados con las teorías anticontagionistas, que consideraban que era relevante estudiar los factores atmosféricos o ambientales que podían incidir en la putrafacción de animales y vegetales. Tal como recuerda Maximiliano Fiquepron (2018), para la década de 1870, no había un acuerdo internacional al respecto sobre el tema, ni siquiera entre los médicos. Como 
veremos, el propio Sarmiento se había pronunciado unos años antes dudando de la solidez de ambas teorías científicas.

Como marco de esta discusión, el vínculo entre meteorología y salud aparece reiteradamente en escritos del siglo XIX (Anderson, 2005; Fleming, 1990; Frisinger, 1977; Jankovic, 2006; Barboza, 2012) y localmente es construido por prácticas de observación meteorológicas llevadas adelante por médicos. La Asociación Médica Bonaerense, a través de su publicación quincenal, la Revista Médica-Quirúrgica, difundía notas asociadas a este vínculo):

Cuando Hipócrates escribía su famoso tratado de aeris et locis ya había comprendido ... que el estudio de la atmósfera y del suelo debía tener una importancia muy grande en la ciencia médica, que él fundaba ... Los que por deber de profesión consagramos todas las fuerzas de nuestra inteligencia al alivio del hombre enfermo, estamos obligados á no desperdiciar auxilio alguno de los que la experiencia nos brinda para el cumplimiento de nuestra misión; y después de haber presenciado y sufrido los estragos de una epidemia asoladora que como tal se relaciona con el estado de la atmósfera, se vuelve todavía más serio nuestro deber de dedicar particular atención à la Meteorología, é instituir una serie de observaciones propias, que con el curso del tiempo nos den la fórmula de las condiciones atmosféricas del país. Algunos individuos particulares por puro amor à la ciencia, se han consagrado en diversas épocas à este estudio experimental: más el resultado de sus observaciones ni ha tenido la notoriedad suficiente para servir à objetos prácticos, ni esas observaciones han sido recogidas quizá con toda la perfección de los medios de que rara vez puede disponer un particular, de donde resulta que ni la industria, ni la higiene, ni la medicina, han podido utilizar los laudables esfuerzos de aquellos (Ballester, 1868c, p.56).

En una de las páginas de la publicación, se aseguraba que "la Asociación MédicoBonaerense es la que está mejor preparada para dar impulsos à estos estudios [meteorológicos], ya por la decidida voluntad que manifiesta para consagrarse al trabajo, ya también por el considerable número de los socios que la forman" (Ballester, 1868c, p.57). Uno de los integrantes de la citada corporación médica, el doctor Evaristo Pineda, se encargaba entonces de realizar observaciones meteorológicas quincenales que luego aparecieron plasmadas en cuadros de la publicación, acompañadas de comentarios que hacían referencia al clima y a las patologías que los facultativos entendían asociadas a los estados del tiempo. Sirvan como ejemplo los comentarios correspondientes a la tabla de la quincena del 8 de septiembre de 1868:

En la quincena que acaba de trascurrir el temporal ha sido, como habitualmente sucede en nuestra ciudad [Buenos Aires], muy variable, pues así como unos días la atmósfera estuvo serena y despejada, en otros se mostró anubarrada, tempestuosa y lluviosa; sucediendo otro tanto con los vientos reinantes, que tan pronto soplaron con dureza como fueron suaves ... Como consecuencia de las vicisitudes atmosféricas apuntadas, han predominado las afecciones catarrales, inflamatorias, reumáticas y nerviosas; observándose en niños y adultos la bronquitis, los catarros bronquiales y laringeos, corizas, neumonías y pleuresías, plurodinias y demás dolores reumáticos, dolores nerviosos, en especial supraorbitarios, calenturas gástricas, tifoideas y disentería (Ballester, 1868b, p.161). 
Establecida en la práctica esta relación entre salud y meteorología en el ámbito local, los factores económicos cobraban relevancia al atender a la problemática de una manera muy específica. La discusión sobre el orden sanitario no fue una cuestión menor para la economía argentina de mediados del siglo XIX, en particular, si se tienen en cuenta los debates en torno a las epidemias sufridas en Buenos Aires durante la presidencia de Sarmiento. Con relación a la fiebre amarilla, la cantidad de actores que estuvieron involucrados en las disputas, así como su nivel de intervención ofrecía una muestra de la relevancia política del tema: las víctimas de la epidemia, los vecinos, las autoridades, los médicos y el poder ejecutivo se vieron entrecruzados en estas historias. La oposición a la política de las autoridades locales estaba organizada y movilizada en torno a estos temas, inclusive con la formación de varias comisiones populares que competían con los organismos de gobierno local e involucraban a vecinos con importantes antecedes políticos y militares (Fiquepron, 2018). Recuerda Galeano (2009, p.112) que:

En medio de esta situación, y de la multiplicación de críticas a la Municipalidad y al Consejo de Higiene, los sectores opositores conformaron una Comisión Popular para reorganizar la lucha contra la epidemia. En realidad, se trataba del mismo grupo de intelectuales, periodistas, médicos y políticos que durante la epidemia de cólera de 1867 habían logrado destituir a la corporación municipal. Aunque no logró demasiado consenso para sus intervenciones, el propio discurso de la Comisión (con su hostilidad sistemática hacia la administración municipal y los médicos del Consejo de Higiene) es testigo de un rechazo bastante generalizado al rol de algunas autoridades durante las epidemias. Es posible distinguir dos formas distintas de resistencia ante las medidas aplicadas. En primer lugar, aparecieron diversos cuestionamientos a las disposiciones policiales que afectaban las libertades económicas. El propio presidente de la Nación, Domingo Faustino Sarmiento, había desatendido - claro que antes de 1871 - un pedido de los médicos para instrumentar cuarentenas de buques en el puerto. Argumentaba que la teoría de los miasmas y las hipótesis científicas de contagio no eran lo suficientemente sólidas como para coartar la libertad de comercio. Similar actitud se adoptó, durante la epidemia de 1871, a propósito de las inspecciones en los saladeros.

En esta coyuntura, en la publicación quincenal de la Revista Médico-Quirúrgica, del 8 de noviembre del 1868, se escribía una columna en la cual se ponía en tela de juicio la toma de decisiones por parte de las autoridades gubernamentales en materia de higiene pública, y en particular sobre la salud y el bienestar de los habitantes de Buenos Aires.

Si las autoridades de un país deben develarse por sus adelantos y progresos con cuanta mayor razón por la salud y la vida de sus moradores; y sin embargo nuestros gobiernos ocupados en los que se ha dado en llamar hacer política; nuestras Legislaturas in nihil faciendum ocupato y la Municipalidad sin encarar con coraje uno de sus principales deberes, han dejado correr los años, y Buenos Aires hoy, con una población cuatro veces mayor que ahora cuarenta años; con esta población acumulada ... Aquí se construyen habitaciones, de las llamadas para negocio, en las que de los menos que se piensa es de las condiciones higiénicas y en un estrecho espacio se hace un considerable número de pequeñas habitaciones, donde viven casi hacinados hombres, mujeres y niños (Ballester, 1868a, p.233). 
En el mismo sentido, para ponderar la importancia de este aspecto y sus ámbitos de influencia, debe recordarse el lugar que la política inmigratoria ocupaba en la segunda mitad del siglo XIX (Onega, 1982; Lattes, 1975). Sarmiento sostenía sus conocidas opiniones de que no era posible esperar el crecimiento y desarrollo solo de la población natural; la inmigración debía contribuir a multiplicar fuerzas. La población argentina necesitaba mezclarse con la "más adelantada" en hábitos y educación, lo cual iba a proporcionar un medio de riqueza en términos de conocimientos, ciencia e industria para el país; para ello era preciso garantizar la situación de los extranjeros, facilitarles el bienestar (Sarmiento, 1994). Es por ello que Scenna (1974. p.430) marca un contraste entre ese discurso político y lo ocurrido con la epidemia:

Si comparamos estos números [entre habitantes argentinos y extranjeros en el año 1871] con los del censo de 1869 - teniendo presente que habían transcurrido tres años entre el censo y la fiebre amarilla, lo que altera los resultados - se obtiene otro dato de interés: la ciudad de Buenos Aires contaba con tantos habitantes argentinos como extranjeros, de modo que el azote fue especialmente severo entre las colectividades inmigradas.

Teniendo en cuenta esta determinación y de aceptarse el balance sobre las investigaciones que refieren al impacto de las epidemias urbanas de la segunda mitad del siglo XIX y las primeras décadas del XX propuesto por Armus (2007), se desprende que el supuesto bienestar de los extranjeros no fue asegurado. Asimismo, la precariedad de las condiciones de vida de amplios sectores de la población inmigrante ponía en evidencia la incidencia de las políticas públicas, sus fallas y las derivaciones conflictivas de las misma (Fiquepron, 2018; Otero, jun. 1998). En definitiva, más allá del efectivo papel del Estado respecto a las políticas de salud de la época - es decir, haya o no contribuido a mitigar las enfermedades de la población inmigrante y combatido la epidemia -, en los discursos de los representantes del Estado el tópico se asoció con la necesidad de impulsar la meteorología evocando el proceso de expansión y consolidación estatal. ${ }^{5}$

Es posible suponer que la atención que Avellaneda le prestaba al problema de las epidemias, al defender la necesidad de estudios meteorológicos, estuviese ligada a la intención de paliar los efectos que la política del poder ejecutivo había tenido frente al mismo problema unos años antes, y que se manifestaba en diversos frentes de disputa (Meik, 2011). Asimismo, parecía un buen ángulo para conseguir fondos ante representantes del Estado más dispuestos a financiar políticas ya aprobadas respecto de la inmigración y la epidemia, antes que a propiciar ayudas económicas destinadas a investigaciones científicas cuyos objetivos ni siquiera se llegaban a considerar.

El proyecto fue finalmente aprobado; se ponía en marcha la Oficina Meteorológica Argentina el $1^{\circ}$ de diciembre de 1872 . La ley destinaba 6.500 pesos por una única vez para la compra de instrumentos y trecientos pesos para la instalación de la oficina; 150 pesos por mes para el sueldo de un calculista y treinta pesos por mes para gastos de oficina.

Se creaba así una nueva institución científica a la que había que proveer de algunos instrumentos comprados por el Estado, pero cuya dirección de investigaciones y red de colaboradores se aseguraba sin costos. Como en el caso del observatorio, la falta de consenso 
no pasaba por una división entre bárbaros que defendían las políticas de Rosas a un lado y pueblos nuevos al otro, como quería Sarmiento en su discurso inaugural. Por otra parte, a pesar de la persistencia de las disputas facciosas, con los opositores, se coincidía en no invertir grandes sumas en las instituciones científicas; los proyectos se terminaban imponiendo subrayando esto y mostrándolos como parte de otras estrategias políticas: educativas y de política internacional en el caso de la astronomía, sanitarias e inmigratorias en el caso de la meteorología.

\section{Consideraciones finales}

En este artículo vimos los debates suscitados en las cámaras parlamentarias entre 1869 y 1872 acerca del financiamiento y creación de dos instituciones científicas: el Observatorio Nacional Argentino y la Oficina Meteorológica Argentina. Mostramos cómo los defensores de financiar estas instituciones adujeron beneficios generales de diverso orden: económicos, educativos, migratorios, higiénicos y ligados a la grandeza nacional. No obstante, los proyectos de ley eran imprecisos respecto a cómo el desarrollo de los conocimientos en dichos espacios redundaría en los provechos que los parlamentarios alegaban retóricamente que tendrían para el país. Frente a la falta de especificidad, se apelaba de manera general a la asociación de los fondos con otras medidas que habían sido aprobadas y puestas en marcha anteriormente, o bien con las políticas educativas, o agrícolas o bien con las sanitarias ligadas a la inmigración.

La vaguedad de los objetivos perseguidos por las instituciones que se creaban no contribuía a despejar la incógnita sobre su utilidad frente a miembros del Parlamento reticentes a la creación de instituciones dedicadas al desarrollo de investigaciones científicas. Quienes se oponían a los proyectos, amparándose en lo difuso de los beneficios aludidos, objetaban que no eran investigaciones urgentes en situaciones de erarios recargados. Luego esos opositores serían tildados por Sarmiento de desatinados defensores de las políticas rosistas y de "la barbarie" en contra de la moderna unidad nacional. No obstante, teniendo en cuenta la historiografía de las últimas décadas en la Argentina, remarcamos que la oposición a Sarmiento en el Parlamento manifestaba tensiones y conflictos facciosos inclusive entre quienes habían sido perseguidos por Rosas años atrás. Asimismo, mostramos que la advertencia sobre los erarios recargados, no parecía un desatino en el Parlamento, de hecho era aceptada e incorporada por el ejecutivo cuando aclaraba que el gasto necesario para crear las instituciones científicas sería mínimo. En el caso del observatorio el tópico fue incorporado durante el debate; en el caso la Oficina Meteorológica, se convirtió en letra del proyecto.

Finalmente, queremos remarcar que estas reiteradas afirmaciones sobre lo exiguo del gasto requerido para los emprendimientos científicos se sostenían respecto a espacios que fueron usados en posteriores discursos como símbolos del esfuerzo del Ejecutivo por la grandeza nacional. Esta tensión reivindica el empeño que hace unos años ponen algunos historiadores de las ciencias por evaluar con atención, en términos cuantitativos y cualitativos, el tipo de recursos y prácticas puestas en funcionamiento en las instituciones científicas locales. Dicho análisis es imprescindible para resituar los discursos inaugurales y los textos de "batalla" política sobre el desarrollo de las ciencias modernas en la Argentina. 


\section{NOTAS}

${ }^{1}$ Parte de los análisis y discusiones sobre los nuevos planteos teóricos y metodológicos y su relación con la historiografía internacional del siglo XX se encuentran en los primeros tres capítulos de Bragoni (2004).

${ }^{2}$ En realidad, el primer observatorio financiado con fondos estatales en América del Sur había sido el de Río de Janeiro, en Brasil, fundado en 1827 (Barboza, 1994, 2012; Barreto, 1986; Gajardo Reyes, 1930; Keenan, 1991; Keenan, Pinto, Álvarez, 1985; Morize, 1987; Videira, 2000).

${ }^{3}$ En 1865, Gould había conocido a Sarmiento en EEUU mientras era ministro plenipotenciario de Bartolomé Mitre (Bernaola, 2001; Gould, 1881, 1885; Hodge, 1971; Chandler, 1896; Chaudet, 1926; Comstock, 1922; SCA, 1979; Marcus, 1899; Rieznik, 2011).

${ }^{4}$ En este caso, como en otros, la precariedad tanto en los recursos materiales como humanos que presentaban las instituciones educativas en la Argentina no se correspondía con la trascendencia de las funciones que le eran asignadas. Entre otros escollos, se destacaban la poca disponibilidad de los recursos materiales, la escasez de profesores y directores idóneos para el desarrollo de la actividad educativa y la falta de equidad en la designación de las partidas presupuestarias para la reparación de los edificios y el pago de los sueldos de los funcionarios dispuestos para los colegios nacionales de las provincias (Bohoslavsky, Soprano, 2010; Escobar, 2010; Schoo, 2014).

${ }^{5}$ Para dimensionar lo no efímero de este tipo de problemáticas, remarquemos que Ramaciotti (2006), que centra su estudio en la Argentina de mediados del siglo XX, al considerar cómo las enfermedades padecidas por la población ejercieron influencia en las decisiones de las autoridades gubernamentales en el momento de gestionar políticas sanitarias así como la repercusión de estas en la imagen pública estatal; también encuentra dos caminos de incidencia ya sea que las políticas estuvieran direccionadas hacia el combate de dichas afecciones "y/o desplegaran diferentes estrategias para ocultarlas ya que la existencia de estas dolencias incidía gravemente en la legitimidad del gobierno de turno" (p.116).

\section{REFERENCIAS}

ANDERSON, Katharine.

Predicting the weather: Victorians and the science of meteorology. Chicago: The University of Chicago Press. 2005.

ARMUS, Diego.

La ciudad impura: salud, tuberculosis y cultura en Buenos Aires, 1870-1950. Buenos Aires: Edhasa. 2007.

BABINI, José.

Historia de la ciencia en la Argentina. Buenos Aires: Solar. 1986.

BABINI, José.

La evolución del pensamiento científico en la Argentina. Buenos Aires: Fragua. 1954.

BALLESTER, Antonio Tristán. La higiene pública. Revista Médico-Quirúrgica de la Asociación Médica Bonaerense, año 5, n.15, p.233. 1868a.

BALLESTER, Antonio Tristán.

Observaciones meteorológicas de la quincena. Revista Médico-Quirúrgica de la Asociación Médica Bonaerense, año 5, n.11, p.161. 1868b.

BALLESTER, Antonio Tristán.

Importancia de la meteorología. Revista MédicoQuirúrgica de la Asociación Médica Bonaerense, año 5, n.4, p.55-56. 1868c.
BARBOZA, Christina Helena.

As viagens do tempo: uma história da meteorologia em meados do século XIX. Rio de Janeiro: e-papers. 2012.

BARBOZA, Christina Helena.

O encontro do rei com Vênus: a trajetória do Observatório do Castelo no ocaso do Império. Niterói: Universidade Federal Fluminense. 1994.

BARRETO, Luis.

A história do Observatório Nacional. Rio de Janeiro: Museu de Astronomia e Ciências Afins. 1986.

BARSKY, Osvaldo; GELMAN, Jorge.

Historia del agro argentino: desde la conquista hasta fines del siglo XX. Buenos Aires: GrijalboMondadori. 2001.

BERNAOLA, Omar.

Enrique Gaviola y el Observatorio Astronómico de Córdoba: su impacto en el desarrollo de la ciencia argentina. Buenos Aires: Saber y Tiempo. 2001.

BOHOSLAVSKY, Ernesto; SOPRANO, Germán. Un Estado con rostro humano: funcionarios e instituciones estatales en Argentina (desde 1880 hasta la actualidad). Buenos Aires: Prometeo; Universidad Nacional de General Sarmiento. 2010. 
BOTANA, Natalio.

Domingo Faustino Sarmiento: una aventura republicana. Buenos Aires: Fondo de Cultura Económica. 1996.

BRAGONI, Beatriz (Comp.).

Microanálisis: ensayos de historiografía argentina. Buenos Aires: Prometeo. 2004.

CHANDLER, Seth.

The life and work of Dr. Gould. Science, v.4, n.103, p.885-890. 1896.

CHAUDET, Enrique.

La evolución de la astronomía durante los últimos cincuenta años, 1872-1922. Buenos Aires:

Sociedad Científica Argentina. 1926.

COMSTOCK, George.

Biographical memoir: Benjamin Apthorp Gould, 1824-1896. National Academy of Sciences, v.17, n.7, p.155-170. 1922.

DIARIO...

Diario de sesiones de la Cámara de Diputados de la Nación. 3 sept. 1869.

DIARIO...

Diario de sesiones de la Cámara de Senadores de la Nación. 14 sept. 1872.

DIARIO...

Diario de sesiones de la Cámara de Senadores de la Nación. 2 oct. 1869.

DIARIO...

Diario de sesiones de la Cámara de Senadores de la Nación. 8 sept. 1869.

ESCOBAR, Nancy.

Los funcionarios de los colegios nacionales: entre la precariedad y los enfrentamientos con las autoridades locales, 1863-1880. In: Jornadas de Investigadores del Departamento de Historia, 7., 2010, Mar del Plata. Anales... Mar del Plata: Universidad Nacional de Mar del Plata. 2010.

FIQUEPRON, Maximiliano.

Lugares, actitudes y momentos durante la peste: representaciones sobre la fiebre amarilla y el cólera en la ciudad de Buenos Aires, 1867-1871. História, Ciências, Saúde-Manguinhos, v.25, n.2, p.335-351. 2018.

FLEMING, James Rodger.

Meteorology in America, 1800-1870. Baltimore:

The Johns Hopkins University Press. 1990.

FRISINGER, Howard.

History of meteorology to 1800. Massachusetts:

Science History Publications. 1977.

GAJARDO REYES, Iván.

El Observatorio Astronómico Nacional de Chile. Revista Astronómica, v.2, n.2, p.67-77. 1930.
GALEANO, Diego.

Médicos y policías durante la epidemia de fiebre amarilla (Buenos Aires, 1871). Salud Colectiva, v.5, n.1, p.107-120. 2009.

GARCÍA, Susana.

Enseñanza científica y cultura académica: la

Universidad de La Plata y las ciencias naturales, 1900-1930. Rosario: Prohistoria. 2010.

GOULD, Benjamin.

Proyecto para la creación de una oficina meteorológica, del 15 de marzo de 1872.

Copiador 3 (20 de mayo de 1878 a 16 de agosto de 1888), p.73. 1888.

GOULD, Benjamin.

Dr. Gould's work at the Cordoba Observatory. Science, v.5, n.119, p.403-405. 1885.

GOULD, Benjamin.

Resultados del Observatorio Nacional Argentino de Córdoba, v.2 (Archivo del Observatorio de Córdoba, Córdoba). 1881.

GOULD, Benjamin.

Informe a Albarracín, ministro de JCIP, del 31 de enero de 1874. Copiador 2 (9 de junio de 1873 a 23 de marzo de 1878), p.114-141. 1878.

GOULD, Benjamin.

Proyecto de ley para establecer un sistema de observaciones meteorológicas en la República Argentina. Buenos Aires: Imprenta Americana. 1872 .

HODGE, John.

Benjamin Aptthorp Gould and the founding of the Argentine National Observatory. The Americas, v.28, n.2, p.152-175. 1971.

JANKOVIC, Vladimir.

The end of classical meteorology, c. 1800. Geological Society, n.256, p.91-99. 1 ene. 2006.

KEENAN, Philip.

The earliest national observatories in Latin America. Journal for the History of Astronomy, v.22, n.67, p.21-31. 1991.

KEENAN, Philip; PINTO, Sonia; ÁLVAREZ, Héctor.

El Observatorio Astronómico Nacional Chile, 1852 1965. Santiago: Universidad de Chile. 1985.

LATTES, Alfredo.

El crecimiento de la población y sus componentes demográficos entre 1870 y 1970. In: Recchini de Lattes, Zulma; Lattes, Alfredo (Ed.). La población de Argentina. Buenos Aires: Cicred. p.29-66. 1975.

MARCUS, Benjamin.

The early presidents of the American Association. Science, v.10, n.225, p.705-713. 1899. 
MEIK, Kindon.

Disease and hygiene in the construction of a nation: the public sphere, public space, and the private domain in Buenos Aires, 1871-1910. Disertación (Doctorado en Filosofía) - Florida International University, Florida. 2011.

MORIZE, Henrique.

Observatório Astronômico: um século de história, 1827-1927. Rio de Janeiro: Museu de Astronomia e Ciências Afins. 1987.

ONEGA, Gladys.

La inmigración en la literatura argentina, 1880-

1910. Buenos Aires: Cedal. 1982.

OTERO, Hernán.

Estadística censal y construcción de la nación: el caso argentino, 1868-1914. Boletín del Instituto de Historia Argentina y Americana "Dr. Emilio Ravignani", n.16-17, p.123-149. jun. 1998.

PALCOS, Alberto.

El Facundo: rasgos de Sarmiento. Buenos Aires: Elevación. 1945.

PAZ, Gustavo.

El gobierno de los 'conspicuos': familia y poder en Jujuy, 1853-1875. In: Sábato, Hilda; Lettieri, Alberto (Comp.). La vida política en Argentina del siglo XIX: armas, votos y voces. Buenos Aires: Fondo de Cultura Económica. p.223-241. 2003.

PODGORNY, Irina.

El sendero del tiempo y de las causas accidentales: los espacios de la prehistoria en la Argentina, 1850-1910. Rosario: Prohistoria. 2009.

PODGORNY, Irina; LOPES, Maria Margaret. El desierto en una vitrina: museos e historia natural en la Argentina, 1810-1890. Ciudad de México: Limusa. 2008.

PYENSON, Lewis.

Cultural imperialism and exact sciences: german expansion overseas 1900-1930. New York: Peter Lang. 1985.
RAMACCIOTTI, Karina.

Política y enfermedades en Buenos Aires, 19461953. Asclepio, v.58, n.2, p.115-138. 2006.

RIEZNIK, Marina.

Los cielos del sur: los observatorios astronómicos de Córdoba y de la Plata, 1870-1920. Rosario: Protohistoria. 2011.

SARMIENTO, Domingo. Argirópolis (1850). Buenos Aires: Secretaría de Cultura de la Nación. 1994.

SARMIENTO, Domingo.

Discurso inaugural del Observatorio Nacional

Argentino (Archivo del Observatorio de Córdoba, Córdoba). 1871.

SCA.

Sociedad Científica Argentina. Evolución de las ciencias en la República Argentina, 1923-1972. t.7: Astronomía. Buenos Aires: SCA. 1979.

SCENNA, Miguel.

Cuando murió Buenos Aires, 1871. Buenos Aires: La Bastilla. 1974.

SCHOO, Susana.

Los colegios nacionales en el período fundacional del sistema educativo argentino: incidencias y variaciones locales (1863-1888). Historia de la Educación, v.15, n.2, p.37-68. 2014.

VIDEIRA, Antonio Augusto Passos.

O Imperial Observatório do Rio de Janeiro e o trânsito de Venus de seis de dezembro de 1882 . Quipu, v.13, n.3, p.291-307. 2000.

WILDER, John.

The Dudley Observatory and the Scientific Council. Albany: Van Benthuysen. 1858. 